\title{
Effect of particle size on solubility, dissolution rate, and oral bioavailability: evaluation using coenzyme $\mathrm{Q}_{10}$ as naked nanocrystals
}

This article was published in the following Dove Press journal:

International Journal of Nanomedicine

9 November 2012

Number of times this article has been viewed

Jiao Sun'

Fan Wang ${ }^{1,2}$

Yue Sui'

Zhennan She'

Wenjun Zhai'

Chunling Wang'

Yihui Deng'

'College of Pharmacy, Shenyang Pharmaceutical University, Shenyang, China; ${ }^{2}$ Beijing Zhijianjinrui Applied

Pharmaceutical Science Inc,

Beijing, China
Correspondence: Yihui Deng

College of Pharmacy, Shenyang

Pharmaceutical University,

Shenyang, China

Tel +862423986316

Fax +862423986316

Email pharmdeng@gmail.com
Abstract: In this paper work, four naked nanocrystals (size range 80-700 nm) were prepared without any surfactant or polymer using the solvent/nonsolvent method. The effects of particle size on their solubility, dissolution, and oral bioavailability were investigated. Solubility and dissolution testing were performed in three types of dissolution medium, and the studies demonstrated that the equilibrium solubilities of coenzyme $Q_{10}$ nanocrystals and bulk drugs were not affected by the dissolution media but the kinetic solubilities were. Kinetic solubility curves and changes in particle size distribution were determined and well explained by the proposed solubilization model for the nanocrystals and bulk drugs. The particle size effect on dissolution was clearly influenced by the diffusion coefficients of the various dissolution media, and the dissolution velocity of coenzyme $\mathrm{Q}_{10}$ increased as particle size decreased. The bioavailability of coenzyme $\mathrm{Q}_{10}$ after oral administration in beagle dogs was improved by reducing the particle size. For $700 \mathrm{~nm}$ nanocrystals, the $\mathrm{AUC}_{0-48}$ was 4.4-fold greater than that for the coarse suspensions, but a further decrease in particle size from $700 \mathrm{~nm}$ to $120 \mathrm{~nm}$ did not contribute to improvement in bioavailability until the particle size was reduced to $80 \mathrm{~nm}$, when bioavailability was increased by 7.3 -fold.

Keywords: particle size, solubility, dissolution, nanocrystal, bioavailability, coenzyme $\mathrm{Q}_{10}$

\section{Introduction}

Since Sucker et al produced nanoparticles in the 1980s, ${ }^{1}$ nanonization has attracted much attention, especially for improving the bioavailability of poorly soluble drugs. ${ }^{2-7}$ For the oral administration of poorly soluble drugs, due to solubility limits and low dissolution velocity, there is a low concentration gradient between the gut and blood vessels, leading to limited transport, which consequently affects oral absorption. Increasing the solubility and dissolution rate is a promising approach. In this context, nanonization, ie, a reduction in particle size so that at least one dimension measures less than $1000 \mathrm{~nm}$, has been developed based on theoretical considerations, such as the Ostwald-Freundlich equation, ${ }^{2,3,5,7,8}$ the Noyes-Whitney equation, ${ }^{2,4,6-8}$ the Prandtl equation, ${ }^{2,5,9}$ and Ostwald ripening. ${ }^{7}$

Among these theories, the Ostwald-Freundlich equation is specific to nanonization as opposed to micronization, and has been used widely in the pharmaceutical industry, although it has attracted some controversy and has not as yet been thoroughly proven. ${ }^{10}$ It is derived from the Kelvin equation, and expresses the dependence of saturation solubility or equilibrium solubility on particle size, ${ }^{10}$ as follows:

$$
\rho v \frac{R T}{M} \ln \frac{S_{r}}{S_{\infty}}=\frac{2 \lambda_{s l}}{r}
$$


where $S_{r}=$ the solubility of particles of radius $r, S_{\infty}=$ the solubility of the solid of a plane surface (or consisting of large particles), $\lambda_{s l}=$ interfacial tension, $M=$ the molar mass, $\rho=$ density of the solid, $v=$ represents the number of moles of ions formed from one mole of electrolyte, $v$ equals 1 for nonelectrolytes, $R=$ the gas constant, $T=$ absolute temperature. This equation is applicable to spherical particles smaller than $1000 \mathrm{~nm}$ in size, especially $200 \mathrm{~nm} .{ }^{11}$ Equation 2 is the Noyes-Whitney equation: ${ }^{12,13}$

$$
\frac{d C}{d t}=\frac{D A}{V h}\left(C_{s}-C_{x}\right)
$$

where $d C / d t=$ dissolution rate, $D=$ diffusion coefficient, $A=$ surface area, $V=$ the volume of the dissolution medium, $C_{s}=$ saturation solubility, $C_{x}=$ the drug concentration of bulk solution, and $h=$ hydrodynamic boundary layer thickness, which is also affected by particle size as expressed by the Prandtl equation. ${ }^{9}$ This indicates that, after reduction of particle size, increased saturation solubility, an enlarged surface area, and a thinner diffusion layer could dramatically increase the dissolution velocity, thereby simultaneously improving bioavailability. ${ }^{14}$ Ostwald ripening, like "a big fish eating a small fish," is a process in which large crystals grow at the expense of smaller ones as a result of differences in solubility.

The particle size effect related to solubility, dissolution, and bioavailability has been documented by many researchers. ${ }^{4,6,15-17}$ However, the preparations used were stabilized by surfactants or polymers, so may not truly reflect the effects of particle size. Until now, investigations of the particle size effect using naked nanocrystals (ie, 100\% drugs without any stabilizer) have rarely been reported.

For nanocrystal systems, obtaining high drug loadings is a challenge. There is a tendency towards precipitation or agglomeration to reduce the enlarged surface area, which usually need to be resisted by adding surfactants or stabilizers, ${ }^{18}$ so that drug loadings of $86 \%$ (weight of itraconazole/total solid weight) for sub-300 nm itraconazole nanoparticles have been considered to be high. ${ }^{19}$ There are only a few studies about the use of naked nanocrystals in the pharmaceutical industry to date, ${ }^{20-24}$ and most of the relevant research has only involved the preparation method. Wang et $\mathrm{al}^{23}$ and Sun et $\mathrm{al}^{24}$ prepared naked nanocrystals of different sizes with coenzyme $\mathrm{Q}_{10}$ using the solvent/nonsolvent method and studied the relationship between particle size and solubility in four ethanol-water solutions. However, there is still no systematic research on naked nanocrystals. Thus, the present study was designed to investigate further the effect of particle size on solubility and dissolution of coenzyme $\mathrm{Q}_{10}$ from another angle. In addition, the relationship between particle size and bioavailability was investigated using beagle dogs, since this is the accepted model for obtaining more stable data about oral administration.

\section{Materials and methods Materials}

Raw coenzyme $\mathrm{Q}_{10}$ material was purchased from Yunnan Chuxiong Sun Pharmaceutical Co, Ltd (Yunnan, China). Coenzyme $Q_{10}$ capsules were obtained from Shanghai Pukang Pharmaceutical Co, Ltd (Shanghai, China). Absolute ethanol $(99.7 \% \mathrm{v} / \mathrm{v})$ and isopropanol $(99.7 \% \mathrm{v} / \mathrm{v})$ were provided by Tianjin Bodi Chemical Holding Co, Ltd (Tianjin, China). Tween 20 was purchased from Tianjin Kermel Chemical Reagent Co, Ltd (Tianjin, China). High-performance liquid chromatography (HPLC) grade methanol was obtained from Jiangsu Hanbon Science and Technology Co, Ltd (Jiangsu, China) and ethanol was obtained from Tianjin Kermel Chemical Reagent Co, Ltd. All other chemicals and reagents were of analytical grade.

\section{Preparation of naked coenzyme $Q_{10}$ nanocrystal and coarse suspensions}

A precipitation method was developed for producing suspensions of naked coenzyme $\mathrm{Q}_{10}$ nanocrystals. Briefly, $30.0 \mathrm{mg}$ and $10.0 \mathrm{mg}$ of coenzyme $\mathrm{Q}_{10}$ were separately dissolved into $3.0 \mathrm{~mL}$ of ethanol in a water bath at $60^{\circ} \mathrm{C}$ as the organic phase. This was then injected into $27.0 \mathrm{~mL}$ of double-distilled water at 14,000 rpm and stirred for 15 seconds (IKAT18 Ultra Turrax $^{\circledR}$, Germany) to obtain suspensions of $120 \mathrm{~nm}$ and $80 \mathrm{~nm}$, respectively. The suspensions of $400 \mathrm{~nm}$ and $700 \mathrm{~nm}$ nanocrystals were prepared by dispersing $27.0 \mathrm{~mL}$ of doubledistilled water into the organic phase in a $50^{\circ} \mathrm{C}$ water bath at $30 \mathrm{~mL}$ per minute and $15 \mathrm{~mL}$ per minute under stirring at $800 \mathrm{rpm}$ and $400 \mathrm{rpm}$, respectively. The nanocrystal suspension of $80 \mathrm{~nm}$ was concentrated by ultrafiltration (Millipore, Bedford, MA). The coarse suspensions were produced by dispersing $30.0 \mathrm{mg}$ of coenzyme $\mathrm{Q}_{10}$ in $30.0 \mathrm{~mL}$ of distilled water containing $10 \% \mathrm{v} / \mathrm{v}$ ethanol. The coenzyme $\mathrm{Q}_{10}$ content of the five suspensions was adjusted to about $1 \mathrm{mg} / \mathrm{mL}$.

\section{Characterization of nanocrystal suspensions}

Particle size distribution and zeta potential

The particle size distribution of the naked nanocrystals was determined by dynamic light scattering using a PSS Nicomp 
380 ZLS equipped with a He-Ne laser source at $632.8 \mathrm{~nm}$ (PSS Nicomp, Santa Barbara, CA) at $23^{\circ} \mathrm{C}$ under an angle of 90 degrees and characterized by intensity-weighted particle size. The suspension sample (about $1 \mathrm{~mL}$ ) was placed inside the sample holder of the particle size analyzer and then determined directly without dilution. Once the required intensity was reached, an analysis was performed to obtain the mean particle size and polydispersity index. The zeta potential was also measured using the PSS Nicomp 380 ZLS.

\section{Transmission electron microscopy}

The samples of different-sized nanocrystal suspensions were diluted with distilled water, pipetted onto collodion filmcoated copper grids with a mesh size of 300, dried using absorbent paper, stained with phosphorus tungsten acid for 4 minutes, and finally dried under ambient conditions. Morphological evaluation of the particles was performed using a transmission electron microscope (JM-1200EX; JEOL, Tokyo, Japan).

\section{Differential scanning calorimetry}

Thermographs of the nanocrystals and bulk drugs in suspension were obtained using a differential scanning calorimetry instrument (1 STARe; Mettler Toledo, Schwerzenbach, Switzerland). Samples equivalent to $20 \mu \mathrm{g}$ of coenzyme $\mathrm{Q}_{10}$ were placed in aluminum pans and heated from $20^{\circ} \mathrm{C}$ to $60^{\circ} \mathrm{C}$ at a scanning rate of $10^{\circ} \mathrm{C}$ per minute under a nitrogen purge of $40 \mathrm{~mL}$ per minute. The results were analyzed using STARe software. The liquid samples were then placed in separate aluminum pans and dried at room temperature for 12 hours to remove the solvent. Thermographs of dried samples were subsequently obtained under the same operating conditions.

\section{Preparation of dissolution media}

Three types of dissolution medium were utilized in this work to investigate the size effect in a series of in vitro dissolution and solubility experiments. Dissolution medium A contained $1.3 \% \mathrm{w} / \mathrm{v}$ Tween 20 , dissolution medium B contained $1.3 \%$ $\mathrm{w} / \mathrm{v}$ Tween 20 and $5.0 \% \mathrm{v} / \mathrm{v}$ isopropanol, and dissolution medium $\mathrm{C}$ contained $1.3 \% \mathrm{w} / \mathrm{v}$ Tween 20 and 10.0\% v/v isopropanol.

\section{Solubility measurement \\ Shaking behavior}

The kinetic solubility of bulk coenzyme $\mathrm{Q}_{10}$ with different amounts of drug content was examined in dissolution medium A, and two types of shaking behavior were investigated. Samples of coenzyme $\mathrm{Q}_{10} 2 \mathrm{mg}, 6 \mathrm{mg}, 10 \mathrm{mg}$, and $20 \mathrm{mg}$ were each dispersed in $60 \mathrm{~mL}$ of dissolution medium, after which $3 \mathrm{~mL}$ of samples were transferred into vials that were capped and sealed with Parafilm ${ }^{\circledR}$. Samples with the same drug content were taped in two directions, ie, horizontally and vertically, to the shaker platform, and then continuously agitated at $100 \mathrm{rpm}$ in a water bath maintained at $25^{\circ} \mathrm{C}$. The amount of drug dissolved was determined at different time points by passing each sample through a $0.1 \mu \mathrm{m}$ Millipore filter and analyzing the drug concentration using reverse-phase high-performance liquid chromatography (HPLC). The measurements were repeated three times.

\section{Solubility of $\mathrm{CoQ}_{10}$ nanocrystals and bulk drugs}

The equilibrium solubility values for the four coenzyme $\mathrm{Q}_{10}$ nanocrystals and bulk drugs in the three types of dissolution medium were determined using the dilution method. Taking dissolution medium $\mathrm{B}$ as an example, $1 \mathrm{~mL}$ of the coenzyme $\mathrm{Q}_{10}$ nanocrystal suspensions of different particle size (drug content equals approximately $1 \mathrm{mg}$ ) was added to $2 \mathrm{~mL}$ of medium (containing 1.95\% w/v Tween 20 and $7.5 \% \mathrm{v} / \mathrm{v}$ isopropanol) in a capped vial to give dissolution medium B (containing 1.3\% w/v Tween 20 and 5.0\% v/v isopropanol). The vial was sealed with Parafilm, taped horizontally to the shaker platform, and then continuously agitated at $100 \mathrm{rpm}$ in a water bath maintained at $25^{\circ} \mathrm{C}$. At different time points, the equilibrated samples were passed through a three-layer membrane filter (pore size from the top to bottom layer being $0.1 \mu \mathrm{m}, 0.05 \mu \mathrm{m}$, and $0.1 \mu \mathrm{m}$ ), while a one-layer $0.05 \mu \mathrm{m}$ Millipore filter was also investigated. The filtrate was analyzed by reversephase HPLC.

\section{In vitro dissolution profile measurement}

Dissolution studies of five coenzyme $\mathrm{Q}_{10}$ suspensions were conducted in a drug dissolution test apparatus (ZRS-8G, Tianda Tianfa Technology Co, Ltd, Tianjin, China) using the paddle method. Based on the solubility values obtained in the previous section, $0.6 \mathrm{~mL}$ of coenzyme $\mathrm{Q}_{10}$ nanocrystal suspension (containing $0.6 \mathrm{mg}$ of coenzyme $\mathrm{Q}_{10}$ ) was dispersed in $900 \mathrm{~mL}$ of dissolution medium to create sink conditions. Dissolution was fixed at $100 \mathrm{rpm}$ and temperature was maintained at $25^{\circ} \mathrm{C}$. At predetermined time intervals, $3 \mathrm{~mL}$ of dissolution medium was withdrawn and replaced with the same volume of fresh medium. A $0.05 \mu \mathrm{m}$ membrane filter was used to remove undissolved drug particles. Drug concentrations were quantified by HPLC and dissolution studies were performed in triplicate. 


\section{Bioavailability studies in beagle dogs}

Twelve healthy beagle dogs (body weight $10.02 \pm 0.57 \mathrm{~kg}$ ) were supplied by the Experimental Animal Center at Shenyang Pharmaceutical University, Shenyang, China. Animal studies were performed in accordance with the guidelines for animal experimentation of Shenyang Pharmaceutical University and approved by the animal ethics committee of the institution. Four different sizes of coenzyme $\mathrm{Q}_{10}$ nanocrystal suspensions, coarse suspensions, and commercial capsules were orally administered to the 12 beagle dogs at a dose of $60 \mathrm{mg} /$ body in a crossover manner. There was a washout period of one week between each consecutive dose. The dogs were fasted for 12 hours before dosing but had free access to water. Blood samples $(1.5 \mathrm{~mL})$ were collected from a antecubital vein into heparinized syringes at 0 (pre-dose), and at $0.17,0.5,1,2,3$, $4,6,8,12,24,36$, and 48 hours after dosing. Serum samples were obtained by centrifugation of the blood samples and stored at $-20^{\circ} \mathrm{C}$ until further analysis.

\section{Analytical method}

\section{$\mathrm{CoQ}_{10}$ in dissolution media}

Samples for solubility and dissolution investigations were subjected to HPLC using a system consisting of an HPLC pump (Model P230; Dalian Elite Analytical Instruments Co, Ltd, Dalian, China) and an ultraviolet detector (Model UV228; Dalian Elite Analytical Instruments Co, Ltd) set at $212 \mathrm{~nm}$. A C18 column (Haito pack ODS C18, $4.6 \mathrm{~mm} \times 100 \mathrm{~mm}, 5 \mu \mathrm{m}$; Zhongshan Haito Biomaterials Technology Ltd, Guangdong Province, China) operated at $30^{\circ} \mathrm{C}$ was used as an analytical column. The mobile phase, a mixture of methanol and ethanol $(40: 60, \mathrm{v} / \mathrm{v})$, was delivered at a flow rate of $1.0 \mathrm{~mL}$ per minute and the injection volume was $100 \mu \mathrm{L}$.

\section{Coenzyme $Q_{10}$ in serum}

Coenzyme $\mathrm{Q}_{10}$ in serum was determined by a validated HPLC method. First, $10 \mu \mathrm{L}$ of internal standard solution (vitamin $\mathrm{K}_{1}, 15 \mu \mathrm{g} / \mathrm{mL}$ in ethanol) was added to $100 \mu \mathrm{L}$ of serum kept for 20 minutes at $37^{\circ} \mathrm{C}$. After mixing thoroughly on a vortex mixer, $200 \mu \mathrm{L}$ of methanol and $600 \mu \mathrm{L}$ of hexane were added to the mixture and mixed well on the vortex mixer for 3 minutes. The mixture was then centrifuged at $10,000 \mathrm{rpm}$ for 10 minutes, and $500 \mu \mathrm{L}$ of supernatant was removed. The extraction was repeated twice in a similar fashion and $600 \mu \mathrm{L}$ of supernatant was removed. The total volume of supernatant $(1100 \mu \mathrm{L})$ was collected and dried under nitrogen. The residue was reconstituted in $100 \mu \mathrm{L}$ of mobile phase which was a mixture of methanol and ethanol
(30:70, v/v), followed by centrifugation at $10,000 \mathrm{rpm}$ for 10 minutes and then determined by HPLC consisting of an HPLC pump and an ultraviolet detector (Model UV200II; Elite) set at $275 \mathrm{~nm}$. A $20 \mu \mathrm{L}$ sample was injected into a C18 column (Hypersil BDS C18, $4.6 \mathrm{~mm} \times 150 \mathrm{~mm}, 5 \mu \mathrm{m}$; Elite) kept at $30^{\circ} \mathrm{C}$ and using the mobile phase for analysis at a flow rate of $1.0 \mathrm{~mL}$ per minute for 10 minutes. All experiments were performed under low lighting conditions.

\section{Pharmacokinetic analysis}

Standard pharmacokinetic parameters for coenzyme $Q_{10}$ were obtained from the plasma concentration-time curves. These pharmacokinetic parameters included peak concentration of the drug in plasma $\left(\mathrm{C}_{\max }\right)$, the time taken to reach peak concentration, and the area under the curve $\left(\mathrm{AUC}_{0 \rightarrow 48}\right)$, which was calculated from 0 to 48 hours using a linear trapezoidal rule.

\section{Statistical analysis}

Statistical analysis was performed using one-way analysis of variance and the Student's $t$-test with SPSS version 16.0 software (IBM Corporation, Armonk, NY). $P$-values less than 0.05 were considered to be statistically significant.

\section{Results and discussion \\ Size distribution and zeta potential of coenzyme $Q_{10}$ nanocrystals \\ in suspension}

As shown in Figure 1, suspensions of the bulk drug and the naked coenzyme $\mathrm{Q}_{10}$ nanocrystals of different sizes had different appearances. The drug contents of the five formulations were the same, and the difference in appearance was caused only by variations in size. It was found that all the nanocrystals were homodispersed, with a shift from a

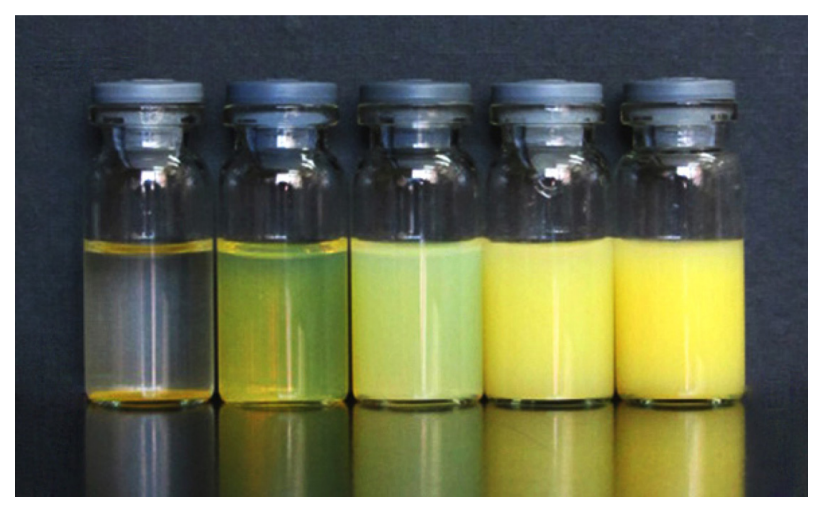

Figure I Suspensions of bulk drugs and naked coenzyme $Q_{10}$ nanocrystals of different sizes after storage for 2 days at room temperature.

Notes: From left to right: bulk drugs, nanocrystals of $80 \mathrm{~nm}, 120 \mathrm{~nm}, 400 \mathrm{~nm}$, and $700 \mathrm{~nm}$, respectively. The drug content of all five formulations was about $1 \mathrm{mg} / \mathrm{mL}$. 
darker to lighter color on reduction in size. In contrast, the bulk drug dispersed in suspension was quite unstable and had sedimented completely by 2 days.

The average size, size distribution, and zeta potential for each of the four different-sized coenzyme $Q_{10}$ nanocrystals are shown in Table 1, and the physical stability results are shown in Figure 2. The average size of the coenzyme $Q_{10}$ nanocrystals in the different suspensions was found to be $80.6 \mathrm{~nm}, 122.2 \mathrm{~nm}, 391.6 \mathrm{~nm}$, and $680.1 \mathrm{~nm}$ (expressed as $80 \mathrm{~nm}, 120 \mathrm{~nm}, 400 \mathrm{~nm}$, and $700 \mathrm{~nm}$, respectively). The $400 \mathrm{~nm}$ - and $700 \mathrm{~nm}$-sized nanocrystals were stable for at least 2 weeks, but the $80 \mathrm{~nm}$ - and $120 \mathrm{~nm}$-sized nanocrystals were physically stable for up to 1 month at room temperature. Zeta potential measurement demonstrated that the nanocrystals in suspension were negatively charged, which might account for the adsorption caused by the large surface area, and contributed to their physical stability. Strong Brownian motion and small density differences between the coenzyme $\mathrm{Q}_{10}$ nanocrystals and the solvent could also have contributed to their stability.

\section{Evaluation of morphology and crystal form}

Transmission electron micrographs clearly show that the nanocrystals were rounded (Figure 3), which is crucial for investigation of the relationship between size and solubility proposed by the classical Ostwald-Freundlich equation.

Differential scanning calorimetry and X-ray powder diffraction analysis are usually used to detect if the crystal form has changed. Because the coenzyme $\mathrm{Q}_{10}$ nanocrystals were prepared without any stabilizer and dispersed in an ethanol-water mixture, additional processes, such as lyophilization or other dry methods, could lead to a change in particle size. Therefore, nanocrystals in suspension were used in subsequent experiments. Small-angle X-ray scattering showed disappointingly low sensitivity for $80 \mathrm{~nm}$ nanocrystals in suspension, so liquid differential scanning calorimetry was chosen to investigate the original suspension sample. The results of liquid differential scanning calorimetry (see Figure 4) show that the nanocrystals failed to demonstrate the melting point of coenzyme $\mathrm{Q}_{10}$, even when concentrated 50-fold. Interestingly, the raw materials dispersed in water had a typical melting point of about $50^{\circ} \mathrm{C}$, which disappeared after 4 hours of storage at room temperature, showing that the crystal transformation of coenzyme $\mathrm{Q}_{10}$ might be induced by the solvent. In order to validate this hypothesis, dried samples were used as a reference preparation. All the samples showed an obvious endothermic peak (see Figure 5), especially for the 50-fold concentrated $120 \mathrm{~nm}$ nanocrystal suspension, in which the amount of drug was high enough to produce sensitivity, and further showed solvent-induced crystal transformation.

\section{Solubility}

For poorly soluble coenzyme $\mathrm{Q}_{10}$, the aqueous solutions provided solubility and dissolution results which were too low for measurement, so the nonionic surfactant Tween 20 was used, in accordance with an already reported method. ${ }^{25}$ Furthermore, different concentrations of isopropanol, which has lower volatility than ethanol, were added to prepare three different media for investigating the effects of size. Although the surfactant was used, it was added after the nanocrystals were prepared so as to develop an appropriate dissolution condition. This is different from the situation in which the surfactant was used as a stabilizer when nanocrystals were prepared. Even though all formulations were prepared using the same concentrations of surfactant, the density of the surfactants dispersed in different-sized nanocrystals was different due to the curvature and surface area.

\section{Investigation of shaking behavior}

In the literature, solubility is always measured by adding an excess amount of drug to the dissolution medium, while shaking continuously in a water bath and sampling at a fixed time (such as 24 hours). ${ }^{4}$ Because determination of solubility at equilibrium takes a long time, and many problems, such as chemical instability, biodegradation, and solvent volatilization, can adversely affect the results, "kinetic" solubility is preferable and is frequently determined.

Table I Particle size, polydispersity index, and zeta potential of coenzyme $Q_{10}$ nanocrystals $(n=4)$

\begin{tabular}{lclr}
\hline Formulation & Particle size $(\mathbf{n m})$ & PI & Zeta potential (mV) \\
\hline A & $80.6 \pm 4.3$ & $0.042 \pm 0.023$ & $-32.20 \pm 0.36$ \\
B & $122.2 \pm 10.5$ & $0.156 \pm 0.005$ & $-31.29 \pm 0.24$ \\
C & $391.6 \pm 14.3$ & $0.238 \pm 0.085$ & $-16.98 \pm 0.27$ \\
D & $680.1 \pm 63.1$ & $0.181 \pm 0.069$ & $-5.58 \pm 1.46$ \\
\hline
\end{tabular}

Abbreviation: $\mathrm{Pl}$, polydispersity index. 


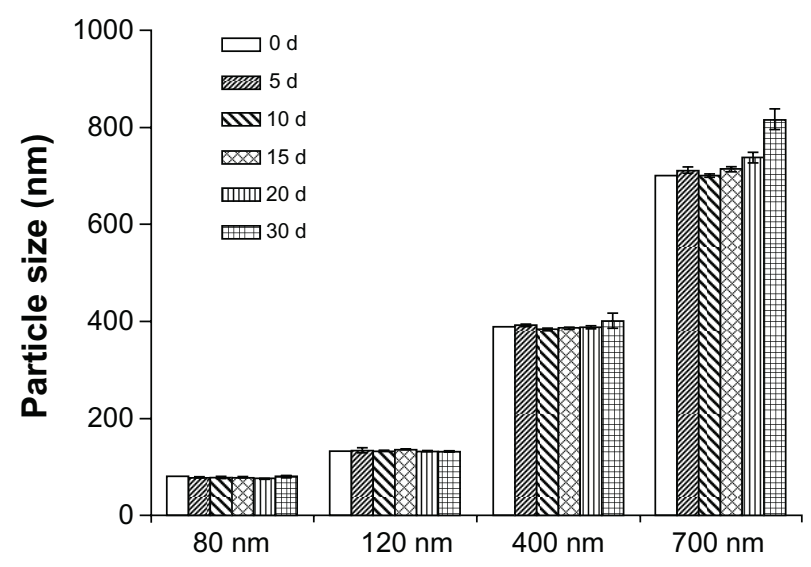

Figure 2 Physical stability of the suspensions of naked coenzyme $Q_{10}$ nanocrystals at room temperature $(n=3)$.

As shown in Figure 6, drug content had obvious effects on kinetic solubility. The higher the drug content, the faster the dissolution rate and the shorter the time taken to reach equilibrium. The shaking behavior did not have a negligible effect. Solubility experiments are usually conducted at $100 \mathrm{rpm}$, but in our study, this rate of agitation was found to be weak and the vibration was distributed nonuniformly
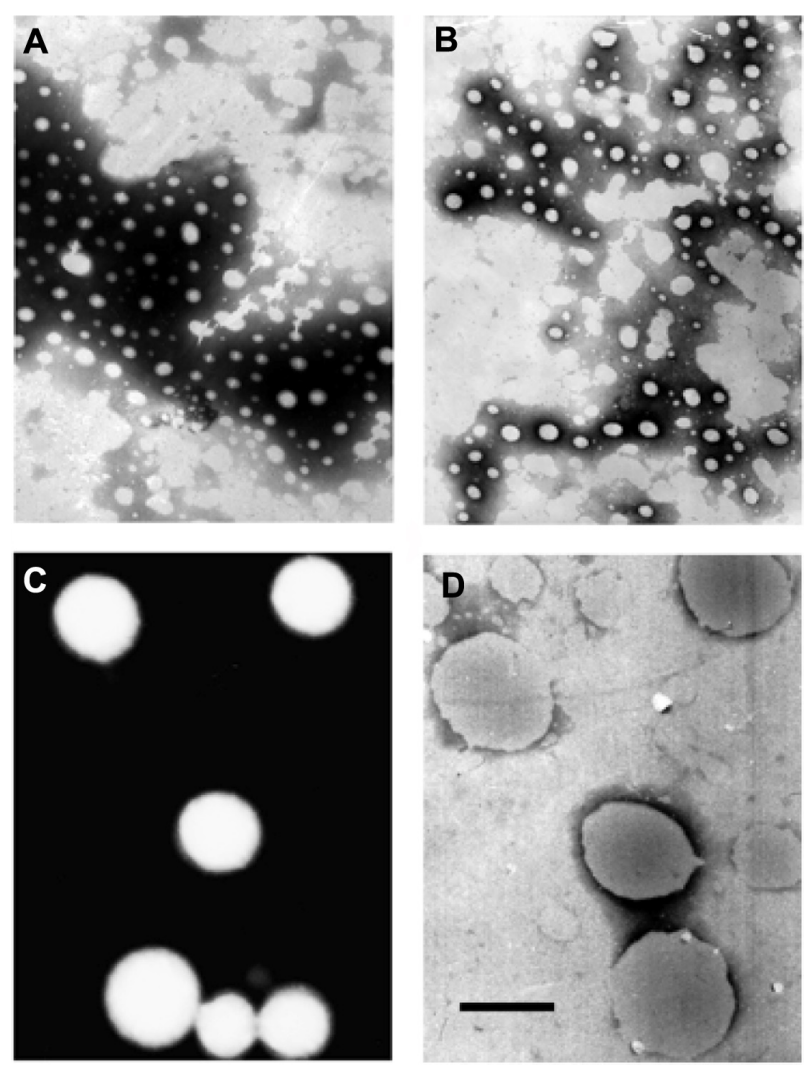

Figure 3 Transmission electron micrographs of coenzyme $Q_{10}$ nanocrystals with particle sizes of (A) $80 \mathrm{~nm}$, (B) $120 \mathrm{~nm}$, (C) $400 \mathrm{~nm}$, and (D) $700 \mathrm{~nm}$. Note: $\mathrm{Bar}=500 \mathrm{~nm}$.

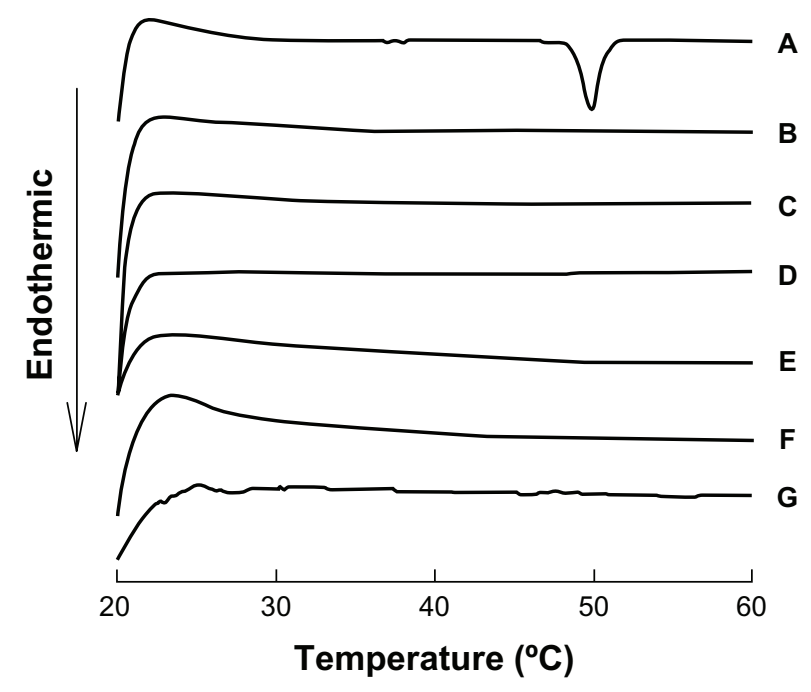

Figure 4 Differential scanning calorimetry patterns of suspensions of coenzyme $Q_{10}$ nanocrystals of different sizes and coarse suspensions.

Notes: (A) Coarse suspensions; (B) $80 \mathrm{~nm}$, (C) $120 \mathrm{~nm}$, (D) $400 \mathrm{~nm}$, (E) $700 \mathrm{~nm}$; (F) coarse suspensions after storage for 4 hours; (G) 50 -fold concentrated $120 \mathrm{~nm}$ nanocrystal suspensions.

when the samples were placed vertically so that drug particles sedimented at the bottom of the tubes. The reduced surface area exposed to the dissolution medium resulted in a longer equilibrium time, as shown in Figure 6. When the samples were placed horizontally, a full vibration effect was obtained and samples with a drug content of $0.10 \mathrm{mg} / \mathrm{mL}, 0.17 \mathrm{mg} / \mathrm{mL}$, and $0.33 \mathrm{mg} / \mathrm{mL}$ all reached equilibrium by day 25 .

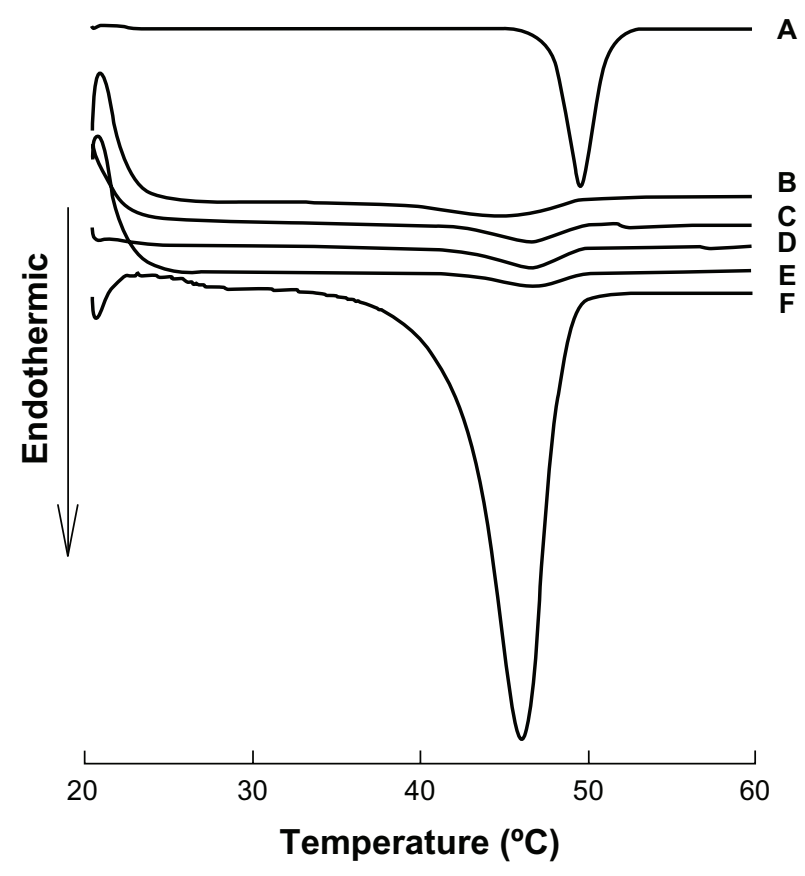

Figure 5 Differential scanning calorimetry patterns of dried suspensions of coenzyme $Q_{10}$ nanocrystals of different sizes and coarse suspensions.

Notes: (A) Coarse suspensions; (B) $80 \mathrm{~nm}$, (C) $120 \mathrm{~nm}$, (D) $400 \mathrm{~nm}$, (E) $700 \mathrm{~nm}$; (F) 50 -fold concentrated $120 \mathrm{~nm}$ nanocrystal suspensions. 


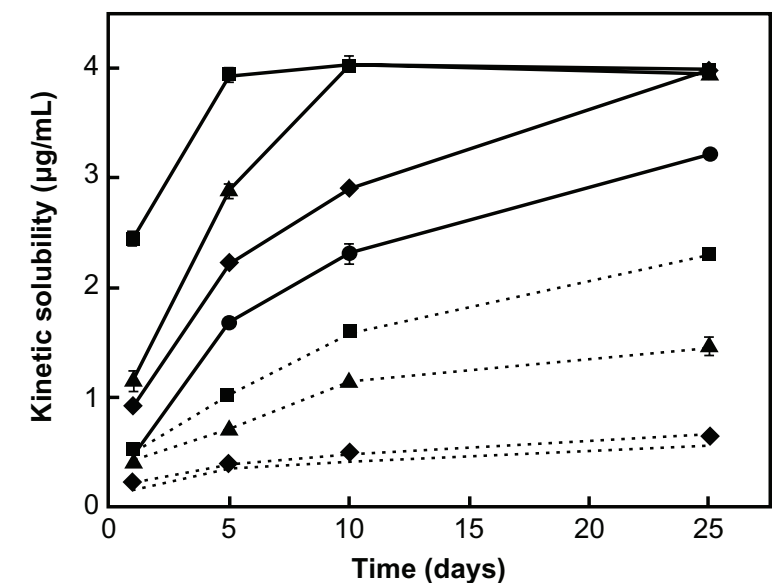

Figure 6 Kinetic solubility of coenzyme $\mathrm{Q}_{10}$ at different drug contents ranging from about $0.03 \mathrm{mg} / \mathrm{mL}$ to $0.33 \mathrm{mg} / \mathrm{mL}$.

Notes: Results are expressed as the mean \pm standard deviation of three replicates. - $=0.03 \mathrm{mg} / \mathrm{mL} ; \boldsymbol{\diamond}=0.10 \mathrm{mg} / \mathrm{mL} ; \boldsymbol{\Delta}=0.17 \mathrm{mg} / \mathrm{mL} ; \boldsymbol{\nabla}=0.33 \mathrm{mg} / \mathrm{mL}$. Solid line: placed horizontally; dotted line: placed vertically.

If equilibrium is not reached, a false impression that solubility is higher as drug content increases is created, leading to inaccuracy when comparing different drugs or formulations. Thus, when kinetic solubility is used as an index for comparison, it is essential to identify the amount of drug being used compared to the vague phrase "excess amount." In addition, the method mentioned above could also be used for assessing equilibrium, and when the solubilities of more than two samples in a series of samples with varying drug content exhibit the same value after full agitation, equilibrium is reached, and the same value is the equilibrium solubility. It is worth noting that this method is not used for ionized drug, the solubility of which is dependent on drug content.

\section{Solubility of coenzyme $Q_{10}$ nanocrystals and bulk drug}

Solubility experiments are usually conducted using drugs in the solid state. Considering that drying naked nanocrystals without any surfactant may change the particle size, and the adsorbed gas as a barrier would influence the dissolution rate, ${ }^{26}$ the suspensions were used directly, ie, by adding a certain volume of nanosuspension or coarse suspension to a solution to obtain the final dissolution medium. In each final dissolution system, about $3.3 \%$ of ethanol residue was introduced by the nanosuspensions. Dialysis in water was initially used to remove ethanol from the nanosuspensions, but this was abandoned because the particle size changed and the particles became unstable. Therefore, each formulation was treated in the same way to ensure consistency. In addition, previous experiments indicated that the influence of 3.3\% ethanol on solubility could be ignored. ${ }^{24}$
As shown in Figure 7, the solubility values obtained using different Millipore filters differed greatly, especially for the $80 \mathrm{~nm}$ and $120 \mathrm{~nm}$ particle sizes. For $80 \mathrm{~nm}$ nanocrystals, after one day in dissolution medium A, the value obtained with the one-layer filter was 25.4-fold higher than that using
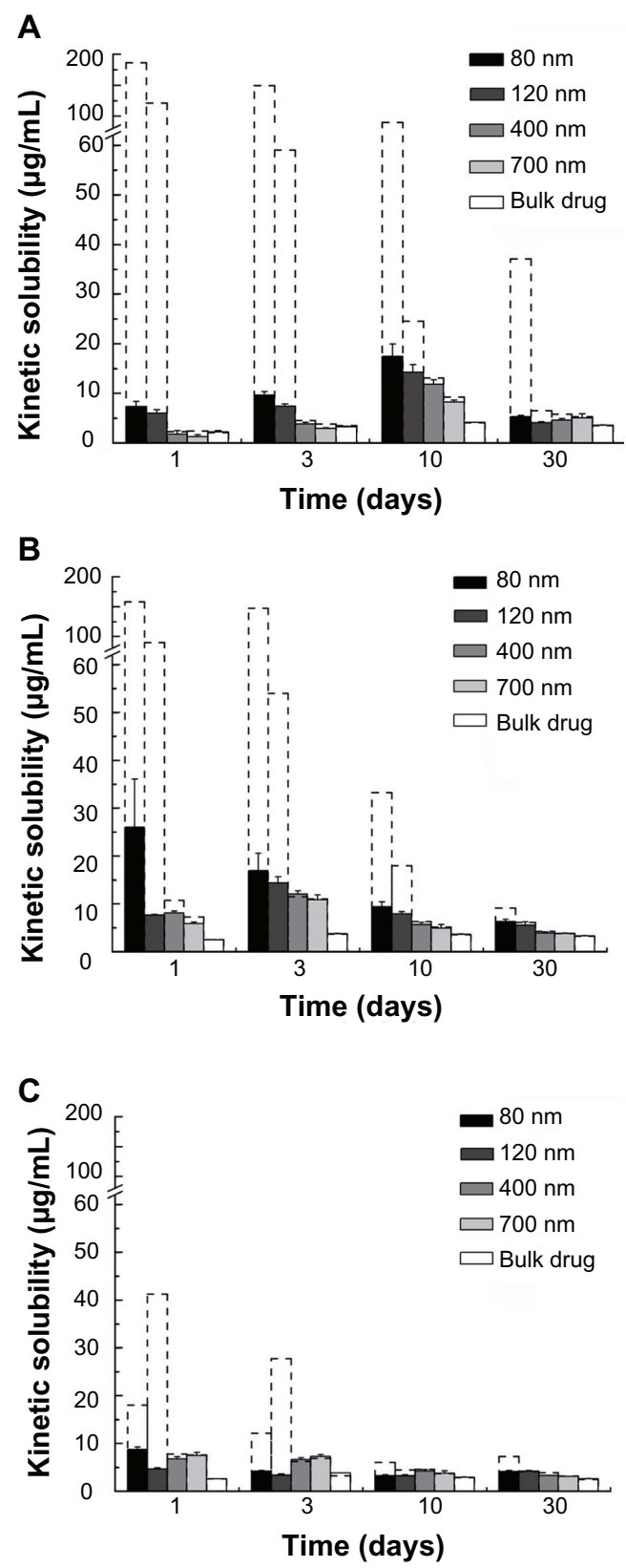

Figure 7 Kinetic solubility of coenzyme $Q_{10}$ nanocrystals and bulk drugs from the suspensions in three types of dissolution medium. Experiments were performed at $100 \mathrm{rpm}$ and $25^{\circ} \mathrm{C}$, and samples were placed horizontally for agitation. The drug content was about $0.33 \mathrm{mg} / \mathrm{mL}$ in every case. The results are expressed as the mean \pm standard deviation of three replicates. (A) Distilled water containing I.3\% w/v Tween 20, (B) distilled water containing $1.3 \% \mathrm{w} / \mathrm{v}$ Tween 20 and $5.0 \% \mathrm{v} / \mathrm{v}$ isopropanol, and (C) distilled water containing $1.3 \% \mathrm{w} / \mathrm{v}$ Tween 20 and $10.0 \% \mathrm{v} / \mathrm{v}$ isopropanol.

Notes: Solid bar: passed through a three-layer membrane filter (the pore size from the top to bottom layer being $0.1 \mu \mathrm{m}, 0.05 \mu \mathrm{m}$, and $0.1 \mu \mathrm{m}$ ); dashed bar: passed through a one-layer $0.05 \mu \mathrm{m}$ Millipore filter. 
the three-layer filter. Although both minimum pore sizes were $0.05 \mu \mathrm{m}$, small particles passed through the one-layer filter more easily. Further reduction of pore size, such as using a $0.03 \mu \mathrm{m}$ filter, was not feasible, because water or ethanol had difficulty passing through. For this reason, a three-layer filter was used for the first time to obtain a better solid-liquid separation. The upper layer trapped large particles and prevented blockages, the middle layer, which is chosen based on the Nicomp distribution analysis intercepted small particles, while the bottom layer acted as a supporting surface, and the crisscrossing of layer to layer provided better solid-liquid separation and accurate solubility data as far as possible. For different sized nanocrystals, the kinetic solubility obtained using three-layer filters increased as the particle size decreased in this experiment.

In a previous study, we proposed a new concept of "interfacial solubility" $\left(C_{i f}\right)$, which is the average concentration of the boundary layer and describes the dissolution process of nanocrystals and bulk drugs. ${ }^{24}$ As shown in Figure 8, "solubilization" is a kinetic process and involves both dissolution and precipitation, which occur at the same time but in different ratios, and usually proceed by diffusion. The driving force is the difference between the interfacial solubility $\left(\mathrm{C}_{i f}\right)$ and the concentration of the bulk solution $\left(\mathrm{C}_{x}\right)$. The disjoining pressure of small particles is greater than that of large particles, so small particles have a higher interfacial solubility. Due to their higher differential concentration, thinner diffusion layer, ${ }^{27}$ and increased surface area, small particles dissolve faster (Figure 8A). For small particles, extremely fast dissolution quickly increases the concentration of the bulk solution to achieve a supersaturated state. On account of the presence of residual particles which induce precipitation and an increasing degree of supersaturation, the rate of precipitation gradually increases until it becomes equal to the rate of dissolution when the bulk solution concentration reaches its maximum, $C_{x(\max )}$. The rate of precipitation then exceeds that of dissolution,

A

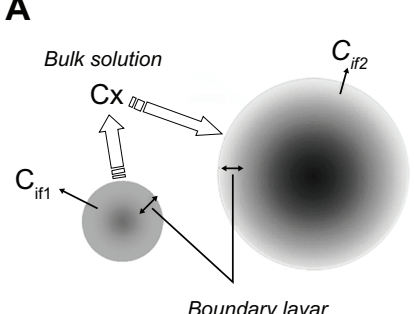

B

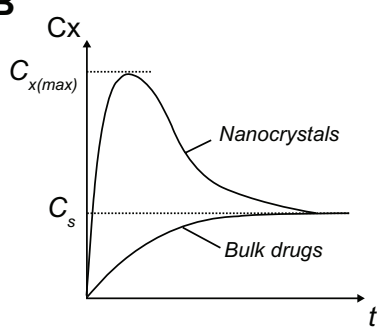

Figure 8 Solubilization model of nanocrystals and bulk drugs. (A) Solubilization process; (B) solubilization curves. the bulk solution concentration decreases, and desaturation increases. As the driving force of precipitation is gradually reduced, the concentration of the bulk solution finally reaches a plateau, which is the constant saturation solubility, $C_{s}$ (ie, the equilibrium solubility). The concentration of the bulk solution $C_{x}$, usually measured in practice, is called the kinetic solubility, ${ }^{8}$ which is a metastable state, and eventually returns to the equilibrium solubility (Figure $8 \mathrm{~B}$ ). This process happens when the concentration gradient is high enough. It happens faster after dilution, so that at last small particles would dissolve and precipitate into large particles, a process known as "Ostwald ripening."

As shown in Figure 7, the content of isopropanol had no obvious effect on the final equilibrium solubility of coenzyme $\mathrm{Q}_{10}$, while the kinetic solubility was different for the three types of dissolution medium, perhaps due to their different diffusion coefficients. As the content of isopropanol in the dissolution medium increased from $0 \%$ to $10 \%$, ripening became faster, and the concentration of the bulk solution reached its maximum at days 10,3 , and 1 , respectively. For the different-sized nanocrystals, the smaller the size, the faster the ripening process (see Table 2). The trend of nanocrystal solubilization was that concentrations first increased to reach a supersaturated state and then decreased. In contrast, the concentrations increased gradually for bulk drugs.

Ripening is a problem when investigating the relationship between particle size and equilibrium solubility in addition to solid-liquid separation, because it is an inevitable process and occurs at the same moment as dilution (as shown in Table 2). When ripening happens, the values obtained represent kinetic solubility rather than equilibrium solubility, and small particles change to large ones when absolute equilibrium is reached. The best method is to determine the equilibrium solubility of coenzyme $Q_{10}$ nanocrystals in suspension directly without dilution, after nanocrystal suspensions of uniform particle size have been prepared. Unfortunately, this is limited by absorption of coenzyme $\mathrm{Q}_{10}$ onto the Millipore filter.

\section{In vitro dissolution study}

In vitro dissolution of coenzyme $\mathrm{Q}_{10}$ from the suspensions containing $80 \mathrm{~nm}, 120 \mathrm{~nm}, 400 \mathrm{~nm}$, or $700 \mathrm{~nm}$ naked nanocrystals and bulk drugs was also investigated in the three types of dissolution medium. Dissolution of nanocrystals smaller than $200 \mathrm{~nm}$ is seldom documented. In this paper, dissolution conditions were selected based on the following considerations: the barrier effect for diffusion caused by dialysis bags, which is a common method for dissolution 
Table 2 Size and size distribution of different coenzyme $Q_{10}$ nanocrystals after dissolution in three types of dissolution medium for different predetermined times*

\begin{tabular}{|c|c|c|c|c|c|c|c|c|c|}
\hline \multirow[t]{2}{*}{ Medium } & \multirow[t]{2}{*}{ Day } & \multicolumn{2}{|l|}{$80 \mathrm{~nm}$} & \multicolumn{2}{|l|}{$120 \mathrm{~nm}$} & \multicolumn{2}{|l|}{$400 \mathrm{~nm}$} & \multicolumn{2}{|l|}{$700 \mathrm{~nm}$} \\
\hline & & Size $(n m)$ & PI & Size $(\mathrm{nm})$ & PI & Size $(n m)$ & PI & Size (nm) & PI \\
\hline & 0 & $78.8 \pm 0.8$ & $0.05 \pm 0.02$ & $134.2 \pm 0.9$ & $0.08 \pm 0.00$ & $376.9 \pm 2.0$ & $0.15 \pm 0.00$ & $698.7 \pm 2.4$ & $0.23 \pm 0.01$ \\
\hline \multirow[t]{3}{*}{ A } & I & $81.1 \pm 3.7$ & $0.07 \pm 0.00$ & $144.9 \pm 4.8$ & $0.06 \pm 0.00$ & $322.9 \pm 4.4$ & $0.18 \pm 0.00$ & $667.2 \pm 5.1$ & $0.23 \pm 0.00$ \\
\hline & 3 & $146.7 \pm 22.8$ & $0.08 \pm 0.01$ & $139.7 \pm 9.3$ & $0.05 \pm 0.01$ & $330.4 \pm 17.0$ & $0.16 \pm 0.00$ & $657.8 \pm 22.9$ & $0.24 \pm 0.01$ \\
\hline & 30 & $x$ & $x$ & $x$ & $x$ & $506.8 \pm 87.0$ & $0.43 \pm 0.06$ & $648.8 \pm 66.3$ & $0.26 \pm 0.03$ \\
\hline \multirow[t]{3}{*}{ B } & I & $104.4 \pm 18.5$ & $0.27 \pm 0.09$ & $189.6 \pm 13.0$ & $0.21 \pm 0.04$ & $385.4 \pm 1.2$ & $0.07 \pm 0.01$ & $688.4 \pm 17.3$ & $0.10 \pm 0.01$ \\
\hline & 3 & $3 \mid 2.8 \pm 74.4$ & $0.39 \pm 0.05$ & $198.0 \pm 43.0$ & $0.30 \pm 0.04$ & $384.4 \pm 39.2$ & $0.18 \pm 0.01$ & $706.1 \pm 79.8$ & $0.24 \pm 0.02$ \\
\hline & 30 & $x$ & $x$ & $x$ & $x$ & $x$ & $x$ & $763.7 \pm|3| . \mid$ & $0.40 \pm 0.10$ \\
\hline \multirow[t]{3}{*}{ C } & I & $x$ & $x$ & $x$ & $x$ & $459.7 \pm 38.2$ & $0.08 \pm 0.01$ & $745.5 \pm 83.2$ & $0.22 \pm 0.04$ \\
\hline & 3 & $x$ & $x$ & $x$ & $x$ & $620.1 \pm 127.3$ & $0.46 \pm 0.08$ & $924.4 \pm 139.7$ & $0.49 \pm 0.19$ \\
\hline & 30 & $x$ & $x$ & $x$ & $x$ & $x$ & $x$ & $x$ & $x$ \\
\hline
\end{tabular}

Notes: *All data were obtained from three experiments and expressed as the mean \pm standard deviation. $x$ represents large visible particles which aggregated into very large bundles and subsequently sedimented.

Abbreviation: PI, polydispersity index.

testing, should be avoided, ${ }^{28}$ the centrifugation method is used extensively for solid-liquid separation, but is not able to separate out small coenzyme $\mathrm{Q}_{10}$ nanocrystals (a suspension of $139 \mathrm{~nm}$ nanocrystals was centrifuged at 30,000 rpm for one hour, and the size distributions of the top, middle, and bottom layers were all the same). There are also reports of using ultracentrifugation or centrifuging twice, ${ }^{29,30}$ but this takes a considerably longer time and is conducted at a low temperature, which changes the drug dissolution conditions; submicron particles, eg, $80 \mathrm{~nm}$, can easily pass through common filters $(\geq 0.1 \mu \mathrm{m}),{ }^{4,31}$ and the three-layer filters described in the previous section are not appropriate for timely sampling. Although in vitro dissolution is often used to predict the in vivo behavior of drugs and experiments are usually carried out at $37^{\circ} \mathrm{C}$, the temperature was set at $25^{\circ} \mathrm{C}$ considering the change in temperature during filtration with a $0.05 \mu \mathrm{m}$ filter and the conditions being consistent with solubility measurement.

As shown in Figure 9, the dissolution rate of coenzyme $\mathrm{Q}_{10}$ in the suspensions was clearly affected by particle size. In dissolution medium A, only the $80 \mathrm{~nm}$ and $120 \mathrm{~nm}$ nanocrystals showed marked dissolution. The peaks observed at 2 hours $(51.4 \%$ and $45.2 \%$ for $80 \mathrm{~nm}$ and $120 \mathrm{~nm}$, respectively) may be due to undissolved small particles passing through the $0.05 \mu \mathrm{m}$ filter. In the case of the $80 \mathrm{~nm}$ nanocrystals, $65.4 \%$ of coenzyme $\mathrm{Q}_{10}$ was dissolved in 24 hours, while for the $120 \mathrm{~nm}$ nanocrystals this value was only $13.2 \%$. In dissolution medium B, the difference in dissolution profiles for the $80 \mathrm{~nm}$ and $120 \mathrm{~nm}$ nanocrystals was further demonstrated. Both showed rapid dissolution in 30 minutes, with the former producing dissolution of $62.8 \%$ and the latter producing dissolution of $37.0 \%$. The
$120 \mathrm{~nm}$ nanocrystals then dissolved slowly, and the fraction only reached $43.3 \%$ in 24 hours. In contrast, the $80 \mathrm{~nm}$ produced a steady increase to $85.4 \%$. Although the $400 \mathrm{~nm}$ and $700 \mathrm{~nm}$ nanocrystals and bulk drugs showed very little dissolution, their dissolution profiles were similar until the ratio of isopropanol increased to $10 \% \mathrm{v} / \mathrm{v}$ (ie, dissolution medium C). Under this condition, the $80 \mathrm{~nm}$ and $120 \mathrm{~nm}$ nanocrystals rapidly dissolved in 30 minutes and both reached $100 \%$ dissolution at 2 hours and 4 hours. The three different dissolution profiles were clearly not influenced by solubility, because the solubilities of coenzyme $Q_{10}$ in the three types of medium were close, as shown in Figure 7. The perfect sink conditions avoided supersaturation and consequent ripening, so that our results only reflect different diffusion behavior. As the content of isopropanol increased, diffusion became easier and dissolution proceeded faster. These findings indicate that the diffusion coefficient of the dissolution medium is an important factor, in addition to the solubility that influenced the particle size effect on dissolution.

\section{Bioavailability study in dogs}

All serum samples were analyzed following the HPLC method described earlier. Calibration curves $(10-1000 \mathrm{ng} / \mathrm{mL}$, eight concentrations) and quality control samples $(50,200$, and $1600 \mathrm{ng} / \mathrm{mL}$ ) were freshly prepared for each analysis. The lower limit of quantification using this method was $10 \mathrm{ng} / \mathrm{mL}$. The linear regression coefficients for the calibration curves ranged from 0.99 to 0.9999 . The accuracy and precision of the quality control samples ranged from $97.3 \%$ to $103.5 \%$ of nominal values, and $1.3 \%$ to $10.6 \%$ for the coefficient of variation, respectively. The results show that 

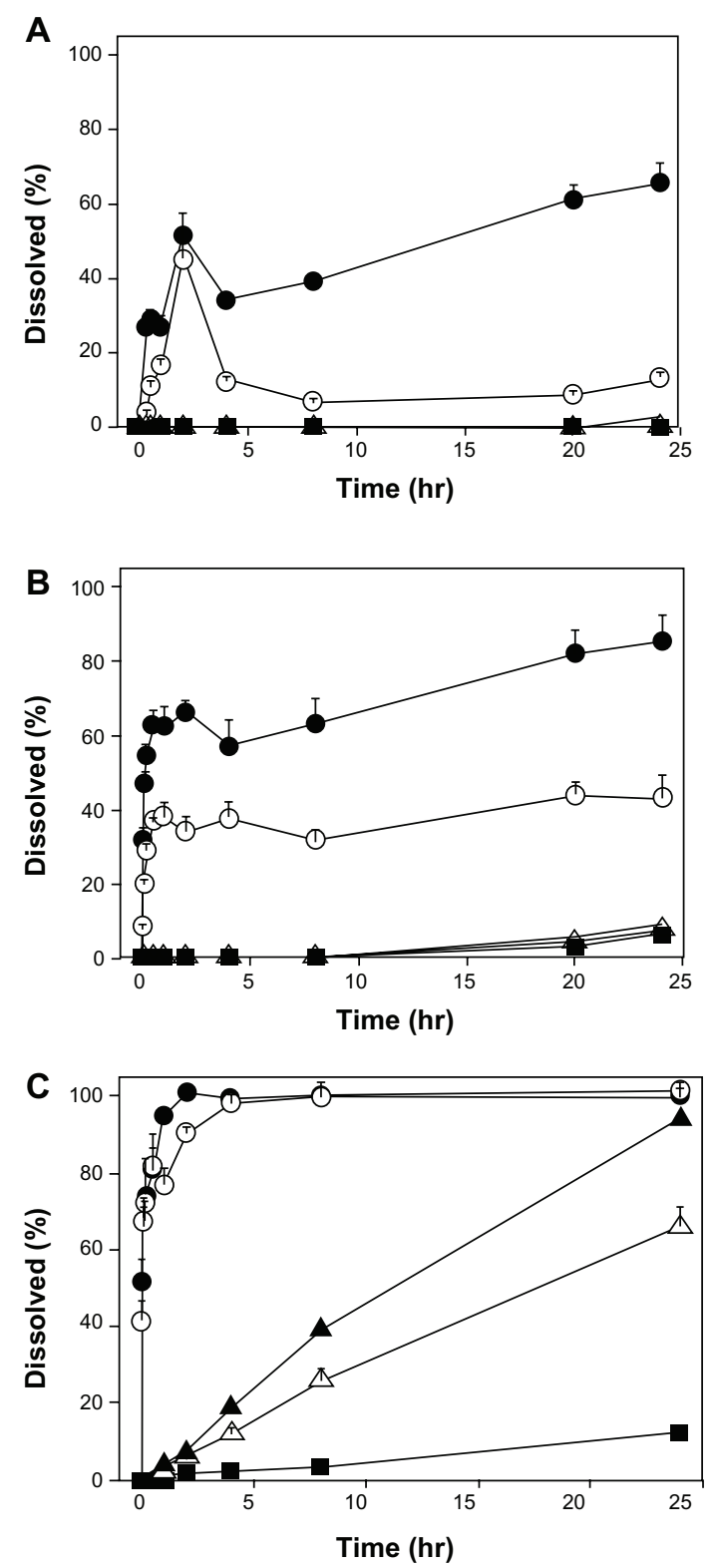

Figure 9 Dissolution profiles of coenzyme $Q_{10}$ nanocrystals and bulk drugs from the suspensions in three types of dissolution medium. (A) Distilled water containing 1.3\% w/v Tween 20, (B) distilled water containing I.3\% w/v Tween 20 and $5.0 \% \mathrm{v} / \mathrm{v}$ isopropanol, and (C) distilled water containing I.3\% w/v Tween 20 and $10.0 \% \mathrm{v} / \mathrm{v}$ isopropanol.

Notes: The dissolution study was performed at $100 \mathrm{rpm}$ and $25^{\circ} \mathrm{C}$. About $0.6 \mathrm{mg}$ of coenzyme $Q_{10}$ was used in $900 \mathrm{~mL}$ of dissolution medium. The results are expressed as the mean \pm standard deviation of three replicates. $\bullet=80 \mathrm{~nm} ; \circ=120 \mathrm{~nm}$; $\Delta=400 \mathrm{~nm} ; \triangle=700 \mathrm{~nm}$ - = bulk drugs.

the method meets the requirements for biological sample analysis.

The serum concentration-time profiles and the pharmacokinetic parameters of coenzyme $\mathrm{Q}_{10}$ following oral administration of different-sized nanocrystal suspensions, coarse suspensions, and capsules in beagle dogs are presented in Figure 10 and Table 3, respectively. These results show that the absorption of coenzyme $\mathrm{Q}_{10}$ markedly

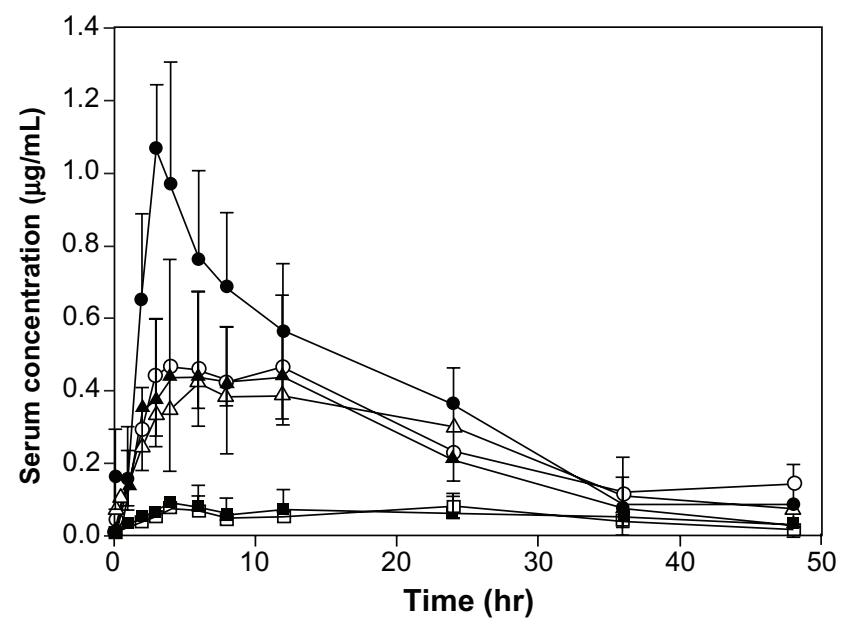

Figure 10 Serum concentration-time profiles of coenzyme $Q_{10}$ after oral administration of different suspensions and capsules at doses of $60 \mathrm{mg} /$ body in beagle dogs.

Notes: Results are expressed as the mean, with the bars showing the standard deviation values. $\bullet=80 \mathrm{~nm}$ nanocrystal suspensions; $\circ=120 \mathrm{~nm}$ nanocrystal suspensions; $\boldsymbol{\Delta}=400 \mathrm{~nm}$ nanocrystal suspensions; $\Delta=700 \mathrm{~nm}$ nanocrystal suspensions; $\boldsymbol{\square}$ = capsules; $\square=$ coarse suspensions ( $\mathrm{n} \geq 4$ ).

improved as the particle size was reduced to the nanoscale range. Compared with coarse suspensions of the bulk drug, the $\mathrm{C}_{\max }$ and $\mathrm{AUC}_{0-48}$ of the naked coenzyme $\mathrm{Q}_{10}$ nanocrystal suspensions with particle sizes of $80 \mathrm{~nm}, 120 \mathrm{~nm}, 400 \mathrm{~nm}$, and $700 \mathrm{~nm}$ were increased by 10.8 -fold, 5.8 -fold, 5.0 -fold, and 4.5 -fold, and by 7.3-fold, 5.1-fold, 4.7-fold, and 4.4fold, respectively. Compared with commercial capsules, the values were increased by 9.2 -fold, 4.9 -fold, 4.2 -fold, and 3.8-fold, and by 6.8 -fold, 4.8-fold, 4.4-fold, and 4.1-fold, respectively. Although the differences in particle size were significant for coenzyme $\mathrm{Q}_{10}$ nanocrystals that were $120 \mathrm{~nm}$ to $700 \mathrm{~nm}$ in size, the increases in $\mathrm{C}_{\max }$ and $\mathrm{AUC}_{0-48}$ observed for these nanocrystal suspensions were almost the same (not statistically significant). This indicates that the particle size effect on oral absorption has a fixed range, and controlling

Table 3 Pharmacokinetic parameters obtained after oral administration of different coenzyme $Q_{10}$ nanocrystal suspensions, coarse suspensions, and capsules $(n \geq 4)^{\mathrm{a}}$

\begin{tabular}{lcll}
\hline Formulation & $\mathbf{T}_{\max }$ (hours) & $\mathbf{C}_{\max }(\mu \mathrm{g} / \mathrm{mL})$ & $\begin{array}{l}\mathrm{AUC}_{0-48} \\
(\mu \mathrm{g} \mathrm{h} / \mathbf{m L})\end{array}$ \\
\hline $80 \mathrm{~nm}$ & $3.25 \pm 0.50^{*}$ & $1.19 \pm 0.18^{* *}$ & $17.52 \pm 1.55^{* * *}$ \\
$120 \mathrm{~nm}$ & $4.86 \pm 3.34^{*}$ & $0.64 \pm 0.24^{* *}$ & $12.18 \pm 5.47^{* *}$ \\
$400 \mathrm{~nm}$ & $5.60 \pm 1.67^{*}$ & $0.55 \pm 0.09 * * *$ & $11.25 \pm 1.16^{* * *}$ \\
$700 \mathrm{~nm}$ & $6.00 \pm 3.58$ & $0.50 \pm 0.06^{* * *}$ & $10.53 \pm 1.79^{* * *}$ \\
Coarse & $19.50 \pm 9.00$ & $0.11 \pm 0.01$ & $2.40 \pm 0.74$ \\
suspension & & & \\
Capsules & $19.60 \pm 12.52$ & $0.13 \pm 0.03$ & $2.56 \pm 0.63$ \\
\hline
\end{tabular}

Notes: aResults are expressed as the mean \pm standard deviation. $* P<0.05$, ${ }^{* *} P<0.01$, and $* * * P<0.001$ compared with the corresponding parameters of coarse suspensions. Abbreviations: $T_{\text {max }}$, time taken to reach peak plasma concentration; $C_{\text {max }}$, peak plasma concentration; AUC, area under the concentration-time curve. 
the sizes in this range could achieve the same bioavailability, which makes application on an industrial scale more feasible. The significant discrepancy between the $80 \mathrm{~nm}$ and $120 \mathrm{~nm}$ nanocrystals in this regard may be attributed mainly to different degrees of uniformity. The $120 \mathrm{~nm}$ nanocrystals displayed a wide distribution (polydispersity index 0.156 , see Table 1) and had two major particle size groups (82 nm and $191 \mathrm{~nm}$, respectively), which led to unstable behavior in vivo which was evident in the pharmacokinetic parameters, with large standard deviations (see Table 3). This unusual result indicates that particle size uniformity is crucial for nanocrystals, because inhomogeneous nanocrystals were not only invalid but also showed increased individual differences.

Because coenzyme $\mathrm{Q}_{10}$ is classified as a Class II drug according to the Biopharmaceutics Classification System, ${ }^{32}$ its absorption could be rate-limited by the dissolution process. Although there was no correlation between in vitro dissolution and in vivo bioavailability, the profiles shown in Figure 9 may still reflect the in vivo dissolution behavior. In the paper published by Jinno et $\mathrm{al},{ }^{4}$ the absorption of cilostazol nanocrystals was lower in the fed state than in the fasted state, with results that were different from those for coarse suspensions. The authors considered that dissolution of cilostazol nanocrystals was rapid enough for absorption to be limited by permeability. The results of this dissolution experiment indicate that lesser absorption of nanocrystals in the fed state than in the fasted state might be partly due to a reduced diffusion coefficient after eating.

The particle size effect was not related to solubility at equilibrium, because the solubility at equilibrium was almost the same for all the four sizes of nanocrystals and the bulk drugs. The improvement in kinetic solubility, dissolution rate, and adhesion ${ }^{18}$ should be responsible for the improved bioavailability of coenzyme $\mathrm{Q}_{10}$. Taken together, reduction in particle size from the microscale to the nanoscale could enhance bioavailability, but a further reduction in particle size did not contribute to the improvement in bioavailability until it was reduced to less than $100 \mathrm{~nm}$.

\section{Conclusion}

Four naked stable coenzyme $\mathrm{Q}_{10}$ nanocrystals of different size were prepared and characterized to generate a drug model for investigating the effects of particle size. The kinetic solubility of four sizes of nanocrystals and bulk drugs was determined in three types of medium. The relationship between size and various solubilities (ie, kinetic solubility, equilibrium solubility) was studied. The results clearly show that particle size was related to kinetic solubility rather than equilibrium solubility. The biggest challenge in investigating the relationship between particle size and equilibrium solubility was found to be solid-liquid separation and the ripening process. A new concept of "interfacial solubility" and a solubilization model of nanocrystals and bulk drugs is proposed, based on the results of this study and is verified by trends in particle size and kinetic solubility. Dissolution testing of four nanocrystals and bulk drugs in three types of dissolution medium also clearly showed the effect of particle size, which was influenced by the diffusion coefficient. The effect of particle size on oral absorption has a fixed range, with coenzyme $Q_{10}$ nanocrystals from $700 \mathrm{~nm}$ to $120 \mathrm{~nm}$ in size having similar bioavailability. Further, for small nanocrystals $120 \mathrm{~nm}$ in size, a wide size distribution (polydispersity index 0.156) increased the individual differences, which indicates that small is not always optimal. Our findings for naked nanocrystals may have significant implications for understanding problems of nanonization, ie, effects of particle size on solubility, dissolution, and in vivo bioavailability, and assist in the design of new nanodrug delivery systems.

\section{Acknowledgment}

This work was supported by the National Basic Research Program of China (973 Program 2009CB 930300).

\section{Disclosure}

The authors report no conflicts of interest in this work.

\section{References}

1. Gassmann P, List M, Schweitzer A, Sucker H. Hydrosols-alternatives for the parenteral application of poorly water soluble drugs. Eur J Pharm Biopharm. 1994;40(2):64-72.

2. Müller RH, Jacobs C, Kayser O. Nanosuspensions as particulate drug formulations in therapy: Rationale for development and what we can expect for the future. Adv Drug Deliv Rev. 2001;47(1):3-19.

3. Dolenc A, Kristl J, Baumgartner S, Planinsek O. Advantages of celecoxib nanosuspension formulation and transformation into tablets. Int J Pharm. 2009;376(122):204-212.

4. Jinno J, Kamada N, Miyake M, et al. Effect of particle size reduction on dissolution and oral absorption of a poorly water-soluble drug, cilostazol, in beagle dogs. J Control Release. 2006;111(1/2):56-64.

5. Müller RH, Peters K. Nanosuspensions for the formulation of poorly soluble drugs: I. Preparation by a size-reduction technique. Int J Pharm. 1998;160(2):229-237.

6. Dong Y, Ng WK, Shen S, Kim S, Tan RBH. Preparation and characterization of spironolactone nanoparticles by antisolvent precipitation. Int J Pharm. 2009;375(1/2):84-88.

7. Kesisoglou F, Panmai S, Wu Y. Nanosizing - oral formulation development and biopharmaceutical evaluation. Adv Drug Deliv Rev. 2007;59(7):631-644.

8. Mauludin R, Müller RH, Keck CM. Kinetic solubility and dissolution velocity of rutin nanocrystals. Eur J Pharm Sci. 2009;36(4/5): $502-510$. 
9. Mosharraf M, Nyström C. The effect of particle size and shape on the surface specific dissolution rate of microsized practically insoluble drugs. Int J Pharm. 1995;122(1/2):35-47.

10. Wu W, Nancollas GH. A new understanding of the relationship between solubility and particle size. J Solution Chem. 1998;27(6): 521-531.

11. Kipp JE. The role of solid nanoparticle technology in the parenteral delivery of poorly water-soluble drugs. Int J Pharm. 2004;284(1/2): 109-122.

12. Noyes AA, Whitney WR. The rate of solution of solid substances in their own solutions. JAm Chem Soc. 1897;19(12):930-934.

13. Dokoumetzidis A, Macheras P. A century of dissolution research: from Noyes and Whitney to the Biopharmaceutics Classification System. Int J Pharm. 2006;321(1):1-11.

14. Babu VR, Areefulla S, Mallikarjun V. Solubility and dissolution enhancement: an overview. J Pharm Res. 2010;3(1):141-145.

15. Ghosh I, Bose S, Vippagunta R, Harmon F. Nanosuspension for improving the bioavailability of a poorly soluble drug and screening of stabilizing agents to inhibit crystal growth. Int J Pharm. 2011;409(1/2): 260-268.

16. Kocbek P, Baumgartner S, Kristl J. Preparation and evaluation of nanosuspensions for enhancing the dissolution of poorly soluble drugs. Int J Pharm. 2006;312(1/2):179-186.

17. Rao GC, Kumar MS, Mathivanan N, Rao ME. Nanosuspensions as the most promising approach in nanoparticulate drug delivery systems. Pharmazie. 2004;59(1):5-9.

18. Rabinow BE. Nanosuspensions in drug delivery. Nat Rev Drug Discov. 2004;3(9):785-796.

19. Matteucci ME, Hotze MA, Johnston KP, Williams RO. Drug nanoparticles by antisolvent precipitation: mixing energy versus surfactant stabilization. Langmuir. 2006;22(21):8951-8959.

20. Zhang JY, Shen ZG, Zhong J, et al. Preparation of amorphous cefuroxime axetil nanoparticles by controlled nanoprecipitation method without surfactants. Int J Pharm. 2006;323(1/2):153-160.

21. Zhang X, Xia Q, Gu N. Preparation of all-trans retinoic acid nanosuspensions using a modified precipitation method. Drug Dev Ind Pharm. 2006;32(7):857-863.
22. Baba K, Pudavar HE, Roy I, et al. New method for delivering a hydrophobic drug for photodynamic therapy using pure nanocrystal form of the drug. Mol Pharm. 2007;4(2):289-297.

23. Wang F, Sun J, Deng YH. Relationship between particle size and solubility of coenzyme $\mathrm{Q}_{10}$ nanosuspensions. J Shenyang Pharm Univ. 2010;27(12):933-939.

24. Sun J, Deng YH, Ma YL, Han J. Equilibrium solubility of naked nanocrystals is not related to particle size. J Shenyang Pharm Univ. 2011;28(08):585-593.

25. Ankola D, Viswanad B, Bhardwaj V, Ramarao P, Kumar M. Development of potent oral nanoparticulate formulation of coenzyme $Q_{10}$ for treatment of hypertension: can the simple nutritional supplements be used as first line therapeutic agents for prophylaxis/therapy? EurJ Pharm Biopharm. 2007;67(2):361-369.

26. Sheridan PL, Buckton G, Storey DE. The extent of errors associated with contact angles II. Factors affecting data obtained using a Wilhelmy plate technique for powders. Int J Pharm. 1994;109(2):155-171.

27. Anderberg EK, Bisrat M, Nyström C. Physicochemical aspects of drug release. VII. The effect of surfactant concentration and drug particle size on solubility and dissolution rate of felodipine, a sparingly soluble drug. Int J Pharm. 1988;47(1/3):67-77.

28. Heng D, Cutler DJ, Chan HK, Yun J, Raper JA. What is a suitable dissolution method for drug nanoparticles? Pharm Res. 2008;25(7): 1696-1701.

29. Van Eerdenbrugh B, Vermant J, Martens JA, et al. Solubility increases associated with crystalline drug nanoparticles: methodologies and significance. Mol Pharm. 2010;16(7):1858-1870.

30. Bergstrom CA, Wassvik CM, Johansson K, Hubatsch I. Poorly soluble marketed drugs display solvation limited solubility. J Med Chem. 2007;50(23):5858-5862.

31. Hecq J, Deleers M, Fanara D, Vranckx H, Amighi K. Preparation and characterization of nanocrystals for solubility and dissolution rate enhancement of nifedipine. Int J Pharm. 2005;299(1/2):167-177.

32. Palamakula A, Soliman M, Khan MM. Regional permeability of coenzyme Q10 in isolated rat gastrointestinal tracts. Pharmazie. 2005;60(3):212-214
International Journal of Nanomedicine

\section{Publish your work in this journal}

The International Journal of Nanomedicine is an international, peerreviewed journal focusing on the application of nanotechnology in diagnostics, therapeutics, and drug delivery systems throughout the biomedical field. This journal is indexed on PubMed Central, MedLine, CAS, SciSearch $\AA$, Current Contents ${ }^{\circledR} /$ Clinical Medicine,

\section{Dovepress}

Journal Citation Reports/Science Edition, EMBase, Scopus and the Elsevier Bibliographic databases. The manuscript management system is completely online and includes a very quick and fair peer-review system, which is all easy to use. Visit http://www.dovepress.com/ testimonials.php to read real quotes from published authors. 